\title{
Cognitive and clinical outcomes of homocysteine-lowering B vitamin treatment in mild cognitive impairment: a randomized controlled trial
}

Celeste A. de $\operatorname{Jager}^{1} *$, Abderrahim Oulhaj ${ }^{1}$, Robin Jacoby ${ }^{2}$, Helga Refsum $^{3,4}$ and A. David $\mathrm{Smith}^{3}$

${ }^{1}$ OPTIMA, Nuffield Department of Clinical Medicine, University of Oxford, Oxford, UK

${ }^{2}$ University Department of Psychiatry, the Warneford Hospital, Oxford, UK

${ }^{3}$ Department of Pharmacology, University of Oxford, UK

${ }^{4}$ Department of Nutrition, Institute of Basic Medical Sciences, University of Oslo, Norway

*These authors contributed equally to the research

Correspondence to: Dr. C. de Jager, OPTIMA, Nuffield Department of Clinical Medicine, John Radcliffe Hospital, Oxford OX3 9DU, UK. E.mail: celeste.de-jager@ndm.ox.ac.uk

Short title: Treatment of mild cognitive impairment

Key words: mild cognitive impairment, homocysteine, folate, cobalamin, pyridoxine, clinical dementia rating, cognitive decline

Words in text body: 3,497

Key points:

- B vitamin treatment over two years slows the decline in cognitive test performance in mild cognitive impairment

- B vitamin treatment over two years leads to apparent improvement in clinical status

- These effects are mainly only found in those with a high baseline level of plasma total homocysteine ( $>11 \mu \mathrm{mol} / \mathrm{L}$ for cognitive tests and $>13 \mu \mathrm{mol} / \mathrm{L}$ for clinical outcomes) 
Background: Homocysteine is a risk factor for Alzheimer's disease. In the first report on the VITACOG trial, we showed that homocysteine-lowering treatment with B vitamins slows the rate of brain atrophy in mild cognitive impairment (MCI). Here we report the effect of B vitamins on cognitive and clinical decline (secondary outcomes) in the same study.

Methods: Double-blind, single centre study. Participants $\geq 70 y$ with MCI randomly assigned to receive a daily dose of $0.8 \mathrm{mg}$ folic acid, $0.5 \mathrm{mg}$ vitamin B12 and $20 \mathrm{mg}$ vitamin $\mathrm{B} 6$ (133 subjects), or placebo (133 subjects), for 2 years. Changes in cognitive or clinical function were analysed by generalized linear models or mixed effects models,

Results: At the end mean plasma total homocysteine was 30 percent lower in those treated with B vitamins relative to placebo. B vitamins stabilized executive function (CLOX) relative to placebo $(\mathrm{P}=0.015)$. There was significant benefit of $\mathrm{B}$ vitamin treatment, among subjects with baseline homocysteine above the median $(11.3 \mu \mathrm{mol} / \mathrm{L})$, in global cognition (MMSE, $\mathrm{P}<0.001$ ), episodic memory (Hopkins Verbal Learning Test-delayed recall, $\mathrm{P}=0.001$ ), and semantic memory (category fluency, $\mathrm{P}=0.037$ ). Clinical benefit occurred in the $\mathrm{B}$ vitamin group for those in the upper quartile of homocysteine at baseline in global CDR $\operatorname{score}(\mathrm{P}=0.02)$ and in IQCODE score $(\mathrm{P}=0.01)$.

Conclusion: In this small intervention trial B vitamins appear to slow cognitive and clinical decline in people with MCI, in particular in those with elevated homocysteine. Further trials are needed to see if this treatment will slow or prevent conversion from MCI to dementia. 


\section{Introduction}

Mild cognitive impairment (MCI) is a syndrome defined as "cognitive decline greater than that expected for an individual's age and education level but that does not interfere notably with activities of daily life" (Gauthier et al., 2006). The prevalence of MCI is about $16 \%$ in those over 70 y old (Graham et al., 1997; Petersen et al., 2009), which means that there are about 5 million in the USA and 14 million in Europe with this condition. Since about half of those with MCI will develop dementia within 5y of diagnosis (Gauthier et al., 2006), there is an urgent need to identify ways of slowing cognitive decline in this sector of the population.

Low levels of B vitamins are associated with cognitive impairment (Selhub et al., 2000; Smith, 2008). The biological pathways involved are probably related to formation of the amino acid homocysteine. If B vitamin levels are lower than normal, total homocysteine (tHcy) levels in plasma become raised. Plasma tHcy levels are known to increase with age (Refsum et al., 2004), and community dwelling elderly with higher than average levels of tHcy perform less well on cognitive tests than those with lower levels (Smith, 2008). Patients with Alzheimer's disease (AD) have higher plasma tHcy than normal elderly people (Clarke et al., 1998; McCaddon et al., 1998; Seshadri et al., 2002; Smith, 2008) and tHcy influences the rate of cognitive decline (Oulhaj et al., 2010). Thus, tHcy is recognised as a risk factor for cognitive impairment and AD.

We have reported the primary outcome of a clinical trial (VITACOG) designed to determine if tHcy-lowering with $\mathrm{B}$ vitamin supplements (folic acid, vitamins $\mathrm{B}_{6}$ and $\mathrm{B}_{12}$ ) over two years would slow the rate of brain atrophy in elderly participants with MCI (Smith et al., 2010). B vitamin treatment reduced average brain atrophy rate by $30 \%$ compared with placebo, and the effect of the treatment was greater the higher the baseline concentration of tHcy; atrophy rate was slowed by $53 \%$ in subjects with tHcy in the upper quartile. The final cognitive test scores were inversely related to the rate of atrophy. In this paper we ask the 
question: are the secondary cognitive and clinical outcomes also influenced by B vitamin treatment, especially in those participants with high baseline tHcy concentrations?

\section{Methods}

The study was carried out under the principles of the Declaration of Helsinki and was approved by National Health Service Oxfordshire Research Ethics Committee A (04/Q1604/100). All participants gave written informed consent. Trial registration: ISRCTN94410159

\section{Study protocol}

The study protocol for this 2-year, placebo-controlled, randomized clinical trial has been described (Smith et al., 2010). Respondents to recruitment advertising ( $\mathrm{n}=646)$ from the Oxford area were screened by telephone for entry criteria and for MCI using a questionnaire, the Telephone Interview for Cognitive Status-modified (TICS-M) (Brandt et al., 1993) ( $\geq 17$ and $\leq 29$ out of 39) and a category fluency test (animals) (Morris et al., 1989). For borderline cases, if TICS-M was $>29$ but category fluency $<19$ or TICS-M word recall $\leq 10 / 20$, then subjects were eligible. Likewise, if TICS-M was $<17$ but category fluency was $\geq 19$ or word recall was $\geq 10 / 20$, subjects were also eligible. Those with MCI who were $\geq 70$ years, had a study partner and had no exclusion criteria (Smith et al., 2010) were invited into the study.

Other measures to confirm the MCI diagnosis (Petersen, 2004) were collected, including the Mini-Mental State Examination (MMSE) (Folstein et al., 1975) ( $\geq 24 / 30)$, a subjective memory complaint with corroboration from a study partner using questions from the Cambridge examination for mental disorders of the elderly (CAMDEX) (Roth et al., 1986) and normal activities of daily living using 5 questions from the Cambridge Behavioural Inventory (Wedderburn et al., 2008). The clinical dementia rating scale (CDR) (Morris, 1993) was assessed, but was not used for MCI classification. Amnestic MCI was not differentiated from non-amnestic MCI in the inclusion criteria. 
At the first clinic visit (baseline), 271 subjects gave written consent and were randomized to treatment or placebo. Centralized telephone randomization was used with full allocation concealment and minimization for age, gender, TICS-M score and MRI consent. Five subjects never started taking the tablets (failed on screening criteria or withdrew consent), leaving 266 subjects that entered the trial. The flow of participants is shown in Figure 1S. Participants, study partners and those assessing outcomes were blind to the assignment of interventions.

The B vitamin group received TrioBe Plus ${ }^{\circledR}$ (Meda AB/Recip AB, Box 906, Pipers väg 2A, SE-170 09 Solna, Sweden), containing $0.8 \mathrm{mg}$ folic acid; $0.5 \mathrm{mg}$ cyanocobalamin; 20 mg pyridoxine $\mathrm{HCl}$. The placebo group received vitamin-free tablets of similar appearance. Both at baseline and at the second visit, blood samples were obtained for routine biochemical tests and assessment of plasma tHcy, folate, vitamin B12, holotranscobalamin (holoTC) and apolipoprotein E (APOE) genotype, as previously described (Smith et al., 2010).

\section{Primary and Secondary Outcome Measures}

The trial was powered for the primary outcome, a change in the rate of brain atrophy over 2 years, and the results have been reported previously (Smith et al., 2010). In that paper, we also reported some secondary outcomes, including compliance by tablet count and biological compliance (change in plasma vitamin status), and evaluation of safety and adverse effects (Smith et al., 2010).

Changes in cognitive and clinical status were secondary outcome measures, and have not been reported previously. At baseline and follow-up, a neuropsychological test battery, described in the trial protocol (Smith et al., 2010), was conducted by trained research nurses and psychologists blind to clinical dementia rating (CDR) and informant information. Tests reported here are representative of particular cognitive domains important in MCI: global cognition (MMSE) (Folstein et al., 1975); episodic memory (Hopkins Verbal Learning Test- 
revised with delayed recall, HVLT-R) (Brandt, 1991); semantic memory (category fluency, CERAD) (Morris et al., 1989); executive function (CLOX) (Royall et al., 1998). The HVLT$\mathrm{R}$ was administered using the 6 different versions consecutively throughout the trial, at baseline, 3, 6, 9, 15, 18 and 24 months, to reduce practice effects. Other tests reported here were only administered at baseline and 24 months. The clinical outcome measures were the global CDR (Morris, 1993) and the short form of the Informant Questionnaire on Cognitive Decline in the Elderly (IQCODE) scores (Jorm, 2004). These tests are described more fully in the Supplementary Appendix.

\section{Statistical analyses}

Efficacy analyses were performed on the intention-to-treat population, defined as participants who were randomly assigned to treatment and received at least one dose of study medication.

The HVLT-delayed recall (DR) score was analysed by a longitudinal method (using data from 5 time points) by logistic regression with Generalized Linear Mixed Model (GLMM, binomial errors, logit link). The HVLT-DR score at 3 months was used as a starting point to reduce the practice effects up to 3 months.

Other neuropsychological tests, for which only baseline and last follow-up measures were collected, were analysed cross-sectionally. These include cognitive tests namely, MMSE, Category fluency and CLOX and clinical measures, namely the CDR and IQCODE. There were fewer clinical measures at follow-up $(\mathrm{N}=191)$ compared with cognitive measures $(n=223)$ due to some study partners being unavailable to complete the CDR and IQCODE.

For each test, rather than analysing the change from baseline to last follow-up, we assessed the effect of treatment by modelling the score at last follow-up (24 months) controlling for its baseline value as well as for potential confounders such as age, gender, education and APOE status. For the CLOX test which has two components, the effect of treatment was assessed by modelling CLOX1 (executive function) at 24 months controlling for CLOX1 at baseline and CLOX2 (praxis element) at 24 months in addition to potential 
confounders described above, as explained in the Supplement (McGuinness et al., 2010). Generalized linear models (GLM) were fitted using different distributions depending on the nature of the outcomes. For the CLOX 1 (ranging from 0 to 15) and MMSE (ranging from 0 to 30 points), we used the Binomial distribution with the logit link. The Category fluency score is the number of correct words given in a timed interval and therefore was analysed using the Poisson distribution and log link. For the IQCODE we used the Gaussian distribution (which corresponds to linear regression). The CDR overall score is an ordered categorical outcome and was recorded as a binary outcome, 0 or $\geq 0.5$, and analysed via logistic regression.

The models were initially fitted without interaction terms to determine the overall effect of treatment on cognition, controlling for covariates including age, APOE $\varepsilon 4$ allele (present or absent), sex and education. Thereafter, pre-specified subgroup analyses were carried out with baseline tHcy included in the interaction term as a binary variable, where study participants were classified as 'low tHcy group' if their baseline tHcy level was below the median $(11.3 \mu \mathrm{mol} / \mathrm{L})$ or 'high tHcy group' for the remainder. For all outcomes, the analysis started with a saturated model, which was then reduced hierarchically using the likelihood-ratio test and the Aikake information criterion (AIC). There was no multiple testing. Statistical analysis was carried out using the R statistical program (www.Rproject.org). Reported $\mathrm{P}$ values are two-sided, and $\mathrm{P}$ values $<0.05$ were considered significant.

\section{Results}

\section{Participants}

Of the 266 subjects starting the intervention, 223 participants $(83.8 \%)$ completed the second visit 2 years later. Reasons for withdrawal have been previously described (Smith et al., 2010) and are summarized in Figure 1S. There was no difference between baseline demographics for the 43 subjects who failed to complete the trial compared with the 223 participants who 
completed the trial (Table 1S). Baseline demographics for the B vitamin treated and placebo groups for the subjects who completed the trial are presented in Tables 1 and 2. There were no significant differences between the groups, except for the depression score.

\section{Biochemical response to treatment}

The baseline and follow-up data for plasma concentrations of tHcy and B vitamins are shown in Table 2. Plasma tHcy increased modestly but significantly in the placebo group, but decreased in the B vitamin group, so that at the end of the trial, there was a nearly $30 \%$ difference between the two groups $(\mathrm{P}<0.001$

\section{Treatment effects on cognitive decline}

Overall effect. Cognitive data at baseline and at the end of trial for all subjects by treatment and by tHcy group are shown in Table 3 as descriptive statistics. The fitted models show no significant overall effect of treatment for MMSE $(\mathrm{P}=0.57)$, HVLT-DR $(\mathrm{P}=0.23)$ or category fluency $(\mathrm{P}=0.92)$. On the other hand, the CLOX test of executive function showed an overall significant difference according to treatment. The odds of a correctly drawn item from CLOX1 at follow-up (24 months), controlling for CLOX2 at follow-up and CLOX1 at baseline as well as for age, education, $A P O E \varepsilon 4$ status and sex, was $30 \%$ higher in B vitamintreated subjects $(\mathrm{P}=0.015)$ relative to placebo.

Subgroup analysis: interaction with homocysteine at baseline. The effect of B vitamin treatment on cognitive outcomes using subgroup analysis defined by the tHcy concentration at baseline gave consistent and significant results.

The final model for HVLT-DR revealed that participants taking placebo in the "high tHcy group' ( $\geq$ median, i.e. $\geq 11.3 \mu \mathrm{mol} / \mathrm{L}$ ) showed significant decline, while participants receiving B vitamins in the 'high tHcy group' showed no significant decline. The average HVLT-DR scores in the 'low tHcy group' did not decline over time for either treatment category (Table 4a). The odds of correctly remembering a word from the list of 12 in the HVLT for a person in the 'high tHcy group' at the end of the trial was $69 \%$ greater if they 
were taking $\mathrm{B}$ vitamins than if they were taking placebo (odds ratio $=1.69, \mathrm{P}=0.001$ ) $($ Table 4a). Figure 1 shows plots of the raw data for the mean HVLT-DR scores for all time points between 3 to 24 months. The plot justifies why we added a quadratic term for time when modelling the log odds ratio by GLMM. It also shows that B vitamin treatment resulted in maintained performance in those with elevated tHcy, while placebo treatment was associated with decline in performance over time.

Table $4 \mathrm{~b}$ summarizes the cross-sectional results for all other tests, assessed at only two time points. For the MMSE, those in the high tHcy group who were treated with B vitamins were 1.58 times more likely to give a correct answer than those receiving placebo $(\mathrm{P}<0.001)$. In the low tHcy group, no significant difference was found in the odds ratio comparing treated and placebo. For category fluency, in 'high tHcy group' the average number of words at follow-up was $9.4 \%$ greater in those on treatment compared with those on placebo $(\mathrm{P}=0.037)$. In the low tHcy group no significant difference was found when comparing treated with placebo. For CLOX1, no interaction with tHcy was found.

\section{Treatment effects on Clinical Outcomes}

Overall effect. In the whole intention-to-treat cohort, there was no significant effect of $\mathrm{B}$ vitamins on $\mathrm{CDR}(\mathrm{P}=0.23)$ or IQCODE $(\mathrm{P}=0.26)$.

Subgroup analysis: interaction with homocysteine at baseline. There was no significant interaction effect when tHcy was categorized by median split for both IQCODE and CDR. But when we compared the upper-quartile of tHcy $(\geq 13.1 \mu \mathrm{mol} / \mathrm{L})$ to the lower quartiles, both IQCODE and CDR showed a significant interaction of baseline tHcy levels with treatment (Table 4b). For the IQCODE, in the high tHcy group, treated subjects had better average IQCODE scores at follow-up compared to placebo (regression coefficient = $0.23 ; \mathrm{P}$-value $=0.011$. In the low tHcy group, no significant difference between treated and placebo was found. 
The distribution of global CDR scores at baseline was almost the same for the placebo $(\mathrm{CDR}=0: 29.8 \% ; \mathrm{CDR}=0.5: 70.2 \% ; \mathrm{CDR}=1: 0 \%)$ and $\mathrm{B}$ vitamin groups $(28.9 \%, 70 \%, 1.1 \%)$. At the end of the trial, the corresponding distribution was: placebo group $(42.1 \%, 54.7 \%$, $3.2 \%)$, B vitamin group $(50 \%, 47.9 \%, 2.1 \%)$. When stratified by tHcy quartiles at baseline, there was a significant effect of treatment on global CDR scores, but only in those in the highest quartile $(\geq 13.1 \mu \mathrm{mol} / \mathrm{L})$, with a shift to a larger proportion having a CDR score of zero (Table 2S and Figure 2). A CDR score of zero was found in $25.0 \%$ of the B vitamintreated group who were in the top quartile of tHcy at baseline and in 58.3\% at follow-up $(\mathrm{P}=0.039$, Fisher exact test). In contrast, those in the high tHcy group taking placebo showed no change in the proportion with CDR of zero from baseline (24\%) to follow-up (28\%, $\mathrm{P}=1.0$ ). The results were not changed when we controlled for covariates including age, sex, ApoE4, and education using logistic regression. In the upper quartile tHcy group the odds of having $\mathrm{CDR}=0$ at follow-up is 5 times greater in the active-treatment group compared to placebo $(\mathrm{P}=0.02$, Table $4 \mathrm{~b})$.

As a secondary analysis, we fitted the GLMM to confirm the GLM results obtained for outcomes with 2 time points (except for CLOX). The results are shown in Figures $2 \mathrm{~S}-4 \mathrm{~S}$ in the Supplement. The beneficial effect of active treatment was significant in each test. Adjusting for other potential confounders, in addition to those above, such as depression score, smoking, diabetes, and systolic blood pressure, did not materially alter the results for all cognitive and clinical outcomes in the GLMM and GLM models (data not shown). 


\section{Discussion}

In the intention-to-treat cohort of subjects with MCI who completed the trial B vitamin treatment did not improve performance in tests of global cognitive function (MMSE), episodic memory (HVLT-DR), or semantic memory (category fluency) or on measures of clinical status (CDR, IQCODE). However, B vitamin treatment did stabilize performance on the CLOX test of executive and planning function and this effect was independent of baseline tHcy, perhaps indicating a direct effect of one or more of the B vitamins.

On the other hand, when analysis was done according to predefined subgroups based on the baseline tHcy concentration, there were clear beneficial effects of B vitamins on tests of episodic memory, semantic memory and global cognition in subjects with baseline tHcy $\geq 11.3 \mu \mathrm{mol} / \mathrm{L}$. In subjects with high baseline tHcy treated with placebo, significant cognitive decline occurred, but this was prevented by treatment with B vitamins.

A similar result was found for the clinical outcomes (CDR and IQCODE), where B vitamin treatment actually improved the clinical outcome but only in subjects with baseline tHcy $\geq 13.1 \mu \mathrm{mol} / \mathrm{L}$. Particularly striking was the effect of B vitamin treatment on the proportion of subjects with a CDR score of zero, which doubled after two years of treatment.

While the sizes of the effects of B vitamin treatment were relatively modest, the fact that they were highly significant and were found in several cognitive domains and also in clinical assessments is consistent with an effect of the intervention on disease progression.

Our results contrast with several negative trials on homocysteine-lowering treatment and cognition or dementia, reviewed by Wald et al. (Wald et al., 2010). The reason may be related to several differences between our trial and previous studies. We included subjects with MCI, who were followed for 2 years, during which the placebo group underwent significant cognitive decline. Several of the trials without treatment effect may have been too short in duration, excluded vitamin B12 or B6 supplements (Wald et al., 2010) or had subjects who were either healthy and therefore did not decline (McMahon et al., 2006; Stott et al., 
2005) or included patients who already suffered from dementia too advanced for an improvement to be readily detected (Aisen et al., 2008). It is noteworthy that in the VITAL trial, subjects with mild $\mathrm{AD}$, but not those with moderately severe $\mathrm{AD}$, showed some improvement upon B vitamin treatment (Aisen et al., 2008).

Our study confirms the positive findings in the FACIT trial (Durga et al., 2007) in which high tHcy $(>13 \mu \mathrm{mol} / \mathrm{L})$ was an inclusion criterion and where there was improvement in several of the test scores, in particular episodic memory, in non-cognitively impaired subjects receiving folic acid. The observations in FACIT and in our trial that improvement was mainly apparent in participants with higher baseline tHcy concentrations may explain the lack of treatment effect in B vitamin trials with low tHcy at baseline. Among our tests, the effect of B vitamin treatment was most striking for episodic memory, where intervention for two years in a subject with a high level of tHcy $>11.3 \mu \mathrm{mol} / \mathrm{L}$ gave a $69 \%$ higher likelihood of correct word recall compared with placebo. Furthermore, there was a significant difference in the rate of decline between the treatment and placebo groups. The same pattern was also observed for MMSE (global) and category fluency (semantic memory) tests. Thus, even though the study was not originally powered for effects on cognitive performance, we have observed significant effects of B vitamin treatment in MCI-relevant cognitive domains in the pre-specified analyses according to baseline tHcy.

The clinical improvement in the CDR suggests a possible reversal of early cognitive impairment in some of those with MCI on B vitamin treatment. Similar to most of the cognitive tests, the effect was only significant for those with raised tHcy levels, in this case in the upper quartile $(\geq 13.1 \mu \mathrm{mol} / \mathrm{L})$. It is striking that a similar threshold was observed for the effect of treatment on the IQCODE, another indicator of clinical status.

A limitation of the trial is the small sample size. As a result we could not investigate other subgroups, e.g., APOE $\varepsilon 4$ status, presence of disease at baseline, drug use etc. which will require larger studies. The compliance was relatively good (Smith et al., 2010) but, 
nevertheless, a per-protocol analysis using biological compliance based on plasma vitamin response may have given even more significant results.

Is homocysteine a causative factor in cognitive decline or just a marker (Obeid and Herrmann, 2006; Smith, 2008)? In this trial population, B vitamin treatment markedly slowed the rate of brain atrophy relative to placebo (Smith et al., 2010). The effect of treatment was highly dependent on plasma tHcy: in those with tHcy in the lowest quartile (i.e., in those with low tHcy), B vitamins had no effect on atrophy rate. In contrast, in those in the top quartile ( $\geq 13.1 \mu \mathrm{mol} / \mathrm{L}$ ) B vitamins had a dramatic effect by halving the rate of atrophy. Furthermore, the rate of atrophy was a major determinant of cognitive function at the end of the trial (Smith et al., 2010). Thus, one interpretation is that lowering tHcy concentrations by administering B vitamins slows brain atrophy, which in turn slows both cognitive and clinical decline. Such an interpretation is consistent with several studies showing that whole brain atrophy rate is strongly correlated with decline in various cognitive measures, including the CDR, in subjects with MCI (Jack et al., 2008). The findings of the VITACOG trial give strong support to the idea of using measures of brain atrophy as endpoints in clinical trials of disease modifying treatments (Jack et al., 2008).

In conclusion, our data indicate that B vitamins may slow cognitive and clinical decline in subjects with MCI, in particular in those who have high tHcy concentrations. 


\section{Acknowledgements}

We wish to thank the participants and their families for taking part in the study. We also thank the entire OPTIMA team who assisted in the conduct of the trial, in particular Ms P. Whitbread (Nurse Study Co-ordinator), Ms E. McCulloch, Mrs Elizabeth King, Ms A. Mullins, Dr A Haigh (research nurses), Ms Carla Martin and Ms Thurza Honey (psychologists), Mrs Carole Johnston, Ms C. Prendergast, and Mr D Warden (laboratory technologists). We are grateful to Mr E. Juszczak and Ms N. Alder for design and implementation of the minimization procedure and to Meda AB/Recip AB, Solna, Sweden, who donated the vitamin and placebo tablets. We warmly thank the following for financial support: Charles Wolfson Charitable Trust, Medical Research Council, Alzheimer's Research Trust, Henry Smith Charity, Thames Valley Dementias and Neurodegenerative Diseases Research Network of the UK National Institute for Health Research, John Coates Charitable Trust and the Sidney and Elizabeth Corob Charitable Trust.

Role of the funding sources

The sponsor (University of Oxford), the funders of the study, and the company providing the tablets had no role in the study design, data collection, data analysis, data interpretation, or writing of the report. The corresponding author had full access to all the data in the study and had final responsibility for the decision to submit for publication.

\section{Conflicts of interest}

A. D. Smith is named as an inventor on two patents held by the University of Oxford on the use of folic acid to treat Alzheimer's disease (US6008221; US6127370); under the University's rules he could benefit financially if the patent is exploited. Drs. Refsum and Smith report having in the past received speaking honoraria from Recip AB, the company that donated the vitamin tablets, and from Axis-Shield, who make the equipment used to assay homocysteine. None of the other authors have any financial disclosures. 


\section{References}

Aisen PS, Schneider LS, Sano M, Diaz-Arrastia R, van Dyck CH, Weiner MF, Bottiglieri T, Jin S, Stokes KT, Thomas RG, et al. 2008. High-dose B vitamin supplementation and cognitive decline in Alzheimer disease: a randomized controlled trial. JAMA 300: 17741783.

Brandt J 1991. The Hopkins Verbal Learning Test: Development of a new memory test with six equivalent forms. Clinical Neuropsychologist 5: 125-142.

Brandt J, Welsh KA, Breitner JC, Folstein MF, Helms M \& Christian JC 1993. Hereditary influences on cognitive functioning in older men. A study of 4000 twin pairs. Arch Neurol 50: 599-603.

Clarke R, Smith AD, Jobst KA, Refsum H, Sutton L \& Ueland PM 1998. Folate, vitamin B12, and serum total homocysteine levels in confirmed Alzheimer disease. Arch Neurol 55: 1449-1455.

Durga J, van Boxtel MP, Schouten EG, Kok FJ, Jolles J, Katan MB \& Verhoef P 2007. Effect of 3-year folic acid supplementation on cognitive function in older adults in the FACIT trial: a randomised, double blind, controlled trial. Lancet 369: 208-216.

Folstein MF, Folstein SE \& McHugh PR 1975. "Mini-mental state". A practical method for grading the cognitive state of patients for the clinician. Journal of Psychiatric Research 12: 189-198.

Gauthier S, Reisberg B, Zaudig M, Petersen RC, Ritchie K, Broich K, Belleville S, Brodaty H, Bennett D, Chertkow H, et al. 2006. Mild cognitive impairment. Lancet 367: 1262 1270.

Graham JE, Rockwood K, Beattie BL, Eastwood R, Gauthier S, Tuokko H \& McDowell I 1997. Prevalence and severity of cognitive impairment with and without dementia in an elderly population. Lancet 349: 1793-1796.

Jack CR, Petersen RC, Grundman M, Jin S, Gamst A, Ward CP, Sencakova D, Doody RS \& Thal LJ 2008. Longitudinal MRI findings from the vitamin E and donepezil treatment study for MCI. Neurobiol Aging 29: 1285-1295.

Jorm AF 2004. The Informant Questionnaire on cognitive decline in the elderly (IQCODE): a review. Int Psychogeriatr 16: 275-293.

McCaddon A 2006. Homocysteine and cognition--a historical perspective. J Alzheimers Dis 9: 361-380.

McCaddon A, Davies G, Hudson P, Tandy S \& Cattell H 1998. Total serum homocysteine in senile dementia of Alzheimer type. Int J Geriatr Psychiatry 13: 235-239. 
McGuinness B, Barrett SL, Craig D, Lawson J \& Passmore AP 2010. Executive functioning in Alzheimer's disease and vascular dementia. Int J Geriatr Psychiatry 25: 562-568.

McMahon JA, Green TJ, Skeaff CM, Knight RG, Mann JI \& Williams SM 2006. A controlled trial of homocysteine lowering and cognitive performance. N Engl J Med 354: 27642772.

Morris JC 1993. The Clinical Dementia Rating (CDR): current version and scoring rules. Neurology 43: 2412-2414.

Morris JC, Heyman A, Mohs RC, Hughes JP, van Belle G, Fillenbaum G, Mellits ED \& Clark C 1989. The Consortium to Establish a Registry for Alzheimer's Disease (CERAD). Part I. Clinical and neuropsychological assessment of Alzheimer's disease. Neurology 39: $1159-1165$.

Obeid R \& Herrmann W 2006. Mechanisms of homocysteine neurotoxicity in neurodegenerative diseases with special reference to dementia. FEBS Lett 580: 29943005 .

Oulhaj A, Refsum H, Beaumont H, Williams J, King E, Jacoby R \& Smith AD 2010. Homocysteine as a predictor of cognitive decline in Alzheimer's disease. Int J Geriatr Psychiatry 25: 82-90.

Petersen RC 2004. Mild cognitive impairment as a diagnostic entity. J Intern Med 256: 183194.

Petersen RC, Roberts RO, Knopman DS, Boeve BF, Geda YE, Ivnik RJ, Smith GE \& Jack CR, Jr. 2009. Mild cognitive impairment: ten years later. Arch Neurol 66: 1447-1455.

Refsum H, Smith AD, Ueland PM, Nexo E, Clarke R, McPartlin J, Johnston C, Engbaek F, Schneede J, McPartlin C, et al. 2004. Facts and recommendations about total homocysteine determinations: an expert opinion. Clin Chem 50: 3-32.

Roth M, Tym E, Mountjoy CQ, Huppert FA \& et al. 1986. CAMDEX: A standardised instrument for the diagnosis of mental disorder in the elderly with special reference to the early detection of dementia. British Journal of Psychiatry 149: 698-709.

Royall DR, Cordes JA \& Polk M 1998. CLOX: an executive clock drawing task. J Neurol Neurosurg Psychiatry 64: 588-594.

Selhub J, Bagley LC, Miller J \& Rosenberg IH 2000. B vitamins, homocysteine, and neurocognitive function in the elderly. Am J Clin Nutr 71: 614S-620S.

Seshadri S, Beiser A, Selhub J, Jacques PF, Rosenberg IH, D'Agostino RB, Wilson PW \& Wolf PA 2002. Plasma homocysteine as a risk factor for dementia and Alzheimer's disease. N Engl J Med 346: 476-483. 
Smith AD 2008. The worldwide challenge of the dementias: a role for B vitamins and homocysteine? Food Nutr Bull 29: S143-172.

Smith AD, Smith SM, de Jager CA, Whitbread P, Johnston C, Agacinski G, Oulhaj A, Jacoby R \& Refsum H 2010. Homocysteine-lowering by B vitamins slows the rate of accelerated brain atrophy in mild cognitive impairment. A randomized controlled trial. PLOS ONE 5: e12244.

Stott DJ, Macintosh G, Lowe GD, Rumley A, McMahon AD, Langhorne P, Tait RC, O'Reilly D S, Spilg EG, Macdonald JB, et al. 2005. Randomized controlled trial of homocysteine-lowering vitamin treatment in elderly patients with vascular disease. Am J Clin Nutr 82: 1320-1326.

Wald DS, Kasturiratne A \& Simmonds M 2010. Effect of folic acid, with or without other B vitamins, on cognitive decline: meta-analysis of randomized trials. Am J Med 123: 522527 e522.

Wedderburn C, Wear H, Brown J, Mason SJ, Barker RA, Hodges J \& Williams-Gray C 2008. The utility of the Cambridge Behavioural Inventory in neurodegenerative disease. $J$ Neurol Neurosurg Psychiatry 79: 500-503. 
Table 1: Baseline characteristics of the study participants.

\begin{tabular}{|c|c|c|c|}
\hline Characteristic & $\begin{array}{l}\text { Placebo group } \\
\qquad(\mathrm{N}=113)\end{array}$ & B Vitamin group $(\mathrm{N}=110)$ & P Value \\
\hline Age at baseline (yr) & $76.7 \pm 4.8$ & $76.8 \pm 5.1$ & 0.81 \\
\hline Female sex & $73(64.6 \%)$ & $70(63.6 \%)$ & 0.88 \\
\hline Total education (yr) & $14.9 \pm 3.5$ & $14.2 \pm 3.5$ & 0.16 \\
\hline$A P O E \& 4$ carrier & $35(31.0 \%)$ & $34(30.9 \%)$ & 0.99 \\
\hline Smoker - ever & $57(50.4 \%)$ & $47(42.7 \%)$ & 0.27 \\
\hline GDS $(0-30) \dagger$ & $7.5 \pm 5.0$ & $5.7 \pm 4.3$ & 0.005 \\
\hline Hemoglobin $(\mathrm{g} / \mathrm{dL})$ & $13.8 \pm 1.2$ & $13.8 \pm 1.2$ & 0.68 \\
\hline $\mathrm{MCV}(\mathrm{fL})$ & $92.9 \pm 4.1$ & $92.5 \pm 4.4$ & 0.44 \\
\hline Diabetes - ever & $11(9.7 \%)$ & $5(4.5 \%)$ & 0.11 \\
\hline $\begin{array}{l}\text { Previous stroke, TIA, MRI } \\
\text { infarct }\end{array}$ & $23(20.3 \%)$ & $17(15.6 \%)$ & 0.36 \\
\hline Previous MI & $9(8.0 \%)$ & $8(7.3 \%)$ & 0.85 \\
\hline Systolic BP (mmHg) & $146 \pm 20$ & $147 \pm 23$ & 0.77 \\
\hline Diastolic BP (mmHg & $80 \pm 11$ & $80 \pm 11$ & 0.92 \\
\hline $\operatorname{BMI}\left(\mathrm{kg} / \mathrm{m}^{2}\right)$ & $26.1 \pm 4.0$ & $25.7 \pm 3.6$ & 0.40 \\
\hline Alcohol (units/week) & $7.1 \pm 8.5$ & $9.1 \pm 9.2$ & 0.11 \\
\hline Creatinine $(\mu \mathrm{mol} / \mathrm{L})$ & $98 \pm 16$ & $96 \pm 17$ & 0.45 \\
\hline Treatment period (yr) & $2.1 \pm 0.1$ & $2.1 \pm 0.1$ & 0.74 \\
\hline TICS-M (0-39) & $24.9 \pm 2.8$ & $24.9 \pm 2.8$ & 0.98 \\
\hline $\mathrm{CDR} \geq 0.5$ & $82(70.2 \%) \S$ & $80(71.1 \%) \S \S$ & 0.98 \\
\hline
\end{tabular}

Data are for the subjects who completed the trial; shown as mean $\pm \mathrm{SD}$ or $\mathrm{N}(\%)$. Student's $t$ test for comparisons of continuous variables and $\mathrm{Chi}^{2}$ for categorical variables. APOE, gene for apolipoprotein E; CDR, global clinical dementia rating; GDS, Geriatric Depression Scale; MCV, mean red cell volume; MI, myocardial infarct; TIA, transient ischemic attack; TICS-M, telephone interview of cognitive status, modified. $†$ GDS: $0-10=$ mild, $11-20=$ moderate, $21-$ $30=$ severe depressive symptoms. $\S 95$ or $\$ \$ 96$ completed the trial with CDR. 
Table 2: Folate and cobalamin markers in plasma before and after $2 \mathrm{y}$ of intervention.

\begin{tabular}{|c|c|c|c|c|c|c|}
\hline \multirow[t]{2}{*}{ Variable } & & \multicolumn{2}{|c|}{$\begin{array}{l}\text { Placebo group } \\
\quad(N=113)\end{array}$} & \multicolumn{2}{|c|}{$\begin{array}{l}\text { B Vitamin group } \\
(\mathrm{N}=\mathbf{1 1 0}) \S\end{array}$} & \multirow[b]{2}{*}{$\mathbf{P}$ value $\dagger$} \\
\hline & & $\begin{array}{l}\text { Geometric } \\
\text { mean }\end{array}$ & $95 \% \mathrm{CI}$ & $\begin{array}{l}\text { Geometric } \\
\text { mean }\end{array}$ & $95 \% \mathrm{CI}$ & \\
\hline \multirow{2}{*}{ tHcy (mol/L) } & Before & 11.6 & $10.9-12.9$ & 11.3 & $10.7-11.9$ & 0.53 \\
\hline & After & 12.4 & $11.8-13.1$ & 8.7 & $8.2-9.1$ & $<0.001$ \\
\hline Pvalue $末$ & & $<0.001$ & & $<0.001$ & & \\
\hline \multirow[t]{2}{*}{ Folate $(\mathrm{nmol} / \mathrm{L})$} & Before & 23.0 & $20.4-26.0$ & 22.6 & $20.0-25.5$ & 0.83 \\
\hline & After & 24.7 & $21.9-27.9$ & 83.8 & $74.3-94.6$ & $<0.001$ \\
\hline P value $\ddagger$ & & 0.35 & & $<0.001$ & & \\
\hline & & & & & & \\
\hline \multirow[t]{2}{*}{ Vitamin $B_{12}(\mathrm{pmol} / \mathrm{L})$} & Before & 324 & $303-347$ & 332 & $310-356$ & 0.62 \\
\hline & After & 348 & $326-373$ & 690 & $644-740$ & $<0.001$ \\
\hline \multirow[t]{2}{*}{ Pvalue } & & 0.021 & & $<0.001$ & & \\
\hline & & 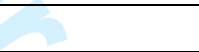 & & & & \\
\hline \multirow[t]{2}{*}{ HoloTC (pmol/L) } & Before & 66 & $59-73$ & 63 & $57-70$ & 0.62 \\
\hline & After & 68 & $62-75$ & 188 & $171-208$ & $<0.001$ \\
\hline Pvalue $\ddagger$ & & 0.34 & & $<0.001$ & & \\
\hline
\end{tabular}

Data are for the subjects who completed the trial. Variables were log transformed prior to analysis. Geometric means and the CI of the means are shown. Active treatment group received daily supplements of folic acid $(0.8 \mathrm{mg})$, vitamin $B_{12}(0.5 \mathrm{mg})$ and vitamin $B_{6}(20 \mathrm{mg})$ for 24 months. Abbreviations: tHcy, plasma total homocysteine; HoloTC, holotranscobalamin. $†$ Student's $t$-test for unpaired samples; $\ddagger$ Student’s $t$-test for paired samples. §The values after treatment refer to 109 subjects since one declined to give a blood sample. 
Table 3: Cognitive test scores at baseline and after $2 \mathrm{y}$ of intervention by median split of homocysteine

\begin{tabular}{|c|c|c|c|c|}
\hline Cognitive Test & $\begin{array}{l}\text { Placebo group } \\
\quad(N=113\end{array}$ & & $\begin{array}{l}\text { B Vitamin group } \\
(N=110)\end{array}$ & \\
\hline & Baseline & Follow-up & Baseline & Follow-up \\
\hline MMSE: $\quad$ Low tHcy & $28.1 \pm 1.6$ & $28.1 \pm 1.9$ & $28.3 \pm 1.8$ & $27.8 \pm 2.4$ \\
\hline High tHcy & $28.4 \pm 1.2$ & $27.2 \pm 2.5$ & $28.2 \pm 1.8$ & $27.9 \pm 2.1$ \\
\hline HVLT DR: Low tHcy & $* 7.4 \pm 2.5$ & $7.9 \pm 3.2$ & $* 7.0 \pm 2.7$ & $7.6 \pm 3.7$ \\
\hline High tHcy & $* 6.9 \pm 2.8$ & $5.9 \pm 3.6$ & $* 6.8 \pm 2.6$ & $7.2 \pm 3.3$ \\
\hline & & & & \\
\hline Cat. fluency: Low tHcy & $21.0 \pm 4.7$ & $21.1 \pm 5.5$ & $21.5 \pm 5.0$ & $20.7 \pm 5.0$ \\
\hline High tHcy & $18.6 \pm 5.1$ & $17.6 \pm 5.6$ & $19.5 \pm 4.5$ & $19.6 \pm 4.7$ \\
\hline Low tHcy & $12.4 \pm 1.9$ & $13.0 \pm 1.7$ & $12.8 \pm 1.6$ & $13.5 \pm 1.7$ \\
\hline High tHcy & $12.1 \pm 2.5$ & $12.3 \pm 2.4$ & $12.3 \pm 2.2$ & $12.6 \pm 1.9$ \\
\hline & & & & \\
\hline Low tHcy & $14.1 \pm 1.0$ & $14.5 \pm 0.7$ & $14.6 \pm 0.6$ & $14.3 \pm 1.1$ \\
\hline High tHcy & $14.0 \pm 1.1$ & $14.1 \pm 1.1$ & $14.3 \pm 0.7$ & $14.1 \pm 1.3$ \\
\hline IQCODE: Low tHcy & $3.2 \pm 0.44$ & $3.1 \pm 0.37$ & $3.2 \pm 0.28$ & $2.9 \pm 0.76$ \\
\hline High tHcy & $3.2 \pm 0.48$ & $3.1 \pm 0.68$ & $3.3 \pm 0.30$ & $3.1 \pm 0.56$ \\
\hline CDR sob: Low tHcy & $0.82 \pm 0.7$ & $0.75 \pm 0.7$ & $0.84 \pm 0.79$ & $0.65 \pm 0.94$ \\
\hline High tHcy & $0.89 \pm 0.7$ & $1.4 \pm 1.6$ & $1.1 \pm 0.87$ & $1.0 \pm 1.3$ \\
\hline
\end{tabular}

Data are for the subjects who completed the trial; Shown as means \pm SD. P values were not derived from this data. 'High tHcy' is defined as at or above the median $(11.3 \mu \mathrm{mol} / \mathrm{L})$ for this cohort and 'Low tHcy' is below the median. *HVLT baseline scores ( 0 month) were excluded in preference of 3 month test scores to avoid the learning effect. CDR sob, CDR sum of boxes 
Table 4a Homocysteine subgroup analysis for the efficacy of B vitamin treatment on longitudinal HVLT-DR shown by the Generalized Linear Mixed effects Model (GLMM)

\begin{tabular}{|l|l|l|l|l|l|}
\hline Cognitive test & Parameter & Estimate & SE & Z-value & P-value \\
\hline \multirow{2}{*}{ HVLT-DR } & Intercept & 0.74 & 0.085 & 10.11 & $<0.001$ \\
\cline { 2 - 6 } (Distribution = Binomial) & Linear term & 0.73 & 0.11 & 6.434 & $<0.001$ \\
\cline { 2 - 6 } & Quadratic term & -0.36 & 0.06 & -5.63 & $<0.001$ \\
\hline & Interaction term & -0.17 & 0.05 & -3.25 & 0.001 \\
\hline
\end{tabular}

The column "Parameter" gives the names of the beta coefficients associated with the linear and quadratic terms for time in the log odds ratio.

Table 4b Homocysteine subgroup analysis for the efficacy of B vitamin treatment on crosssectional test performance shown by Generalized Linear Model (GLM)

\begin{tabular}{|c|c|c|c|c|c|}
\hline Cognitive test & Parameter & Estimate & SE & Z- & P-value \\
\hline \multirow{4}{*}{$\begin{array}{l}\text { MMSE at last visit } \\
\text { (Distribution = Binomial) }\end{array}$} & Intercept & 1.61 & 0.20 & 8.0 & $<0.001$ \\
\hline & Treatment & 0.46 & 0.13 & 3.4 & $<0.001$ \\
\hline & Baseline tHcy & 0.49 & 0.14 & 3.6 & $<0.001$ \\
\hline & Treatment_x_tHcy & -0.69 & 0.19 & -3.6 & $<0.001$ \\
\hline \multirow{4}{*}{$\begin{array}{l}\text { Category Fluency at last visit } \\
\text { (Distribution = Poisson) }\end{array}$} & Intercept & 3.07 & 0.08 & 40.3 & $<0.001$ \\
\hline & Treatment & 0.09 & 0.04 & 2.1 & 0.037 \\
\hline & Baseline tHcy & 0.10 & 0.04 & 2.3 & 0.023 \\
\hline & Treatment_x_tHcy & -0.13 & 0.06 & -2.2 & 0.030 \\
\hline \multirow{4}{*}{$\begin{array}{l}\text { Clinical dementia rating }(\mathrm{CDR}) \text { at last } \\
\text { visit } \\
\text { (Distribution = Binomial) }\end{array}$} & Intercept & 0.84 & 0.58 & 1.46 & 0.14 \\
\hline & Treatment & 1.62 & 0.70 & 2.32 & 0.02 \\
\hline & Baseline tHcy & 0.66 & 0.57 & 1.17 & 0.240 \\
\hline & Treatment_x_tHcy & -1.62 & 0.79 & -2.06 & 0.039 \\
\hline \multirow{4}{*}{$\begin{array}{l}\text { IQCODE at last visit } \\
\text { (Distribution = Gaussian) }\end{array}$} & Intercept & 1.50 & 0.23 & 6.40 & $<0.001$ \\
\hline & Treatment & -0.22 & 0.08 & -2.57 & 0.011 \\
\hline & Baseline tHcy & -0.10 & 0.07 & -1.45 & 0.150 \\
\hline & Treatment_x_tHcy & 0.23 & 0.10 & 2.34 & 0.020 \\
\hline \multirow{4}{*}{$\begin{array}{l}\text { CLOX1 } \\
\text { at follow-up }\end{array}$} & Intercept & -5.47 & 0.62 & -8.8 & $<0.001$ \\
\hline & Treatment & 0.26 & 0.11 & 2.4 & 0.015 \\
\hline & Clox 1_baseline & 0.13 & 0.02 & 5.7 & $<0.001$ \\
\hline & Clox2_24months & 0.37 & 0.04 & 8.8 & $<0.001$ \\
\hline
\end{tabular}

This table shows those cognitive outcomes where there was only one follow-up observation (24 months). Treatment category: 0, placebo; 1, B vitamins. Baseline tHcy: 0, high; 1, low. High tHcy is defined as $\geq$ median $(11.2 \mu \mathrm{mol} / \mathrm{L})$ for the cognitive tests and $\geq 13.1 \mu \mathrm{mol} / \mathrm{L}$ for CDR and IQCODE. Adjusted for age, sex, $A P O E \varepsilon 4$ status and education. For more details see Supplement. 
Figure 1 Effect of B vitamin treatment on a test of episodic memory.

The graphs illustrate the longitudinal effect of treatment on unadjusted mean HVLT-DR scores and its interaction with baseline tHcy level. 'Low' and 'High' baseline tHcy refer to values below and above the median $(11.3 \mu \mathrm{mol} / \mathrm{L})$, respectively. $* \mathrm{P}=0.001$ compared with placebo by logistic regression using GLMM (Table 4a).

Figure 2 Effect of B vitamin treatment on the proportion of participants with a CDR score of zero. Low' and 'High' baseline tHcy refer to values below and above the upper quartile (13.1 $\mu \mathrm{mol} / \mathrm{L})$, respectively. 


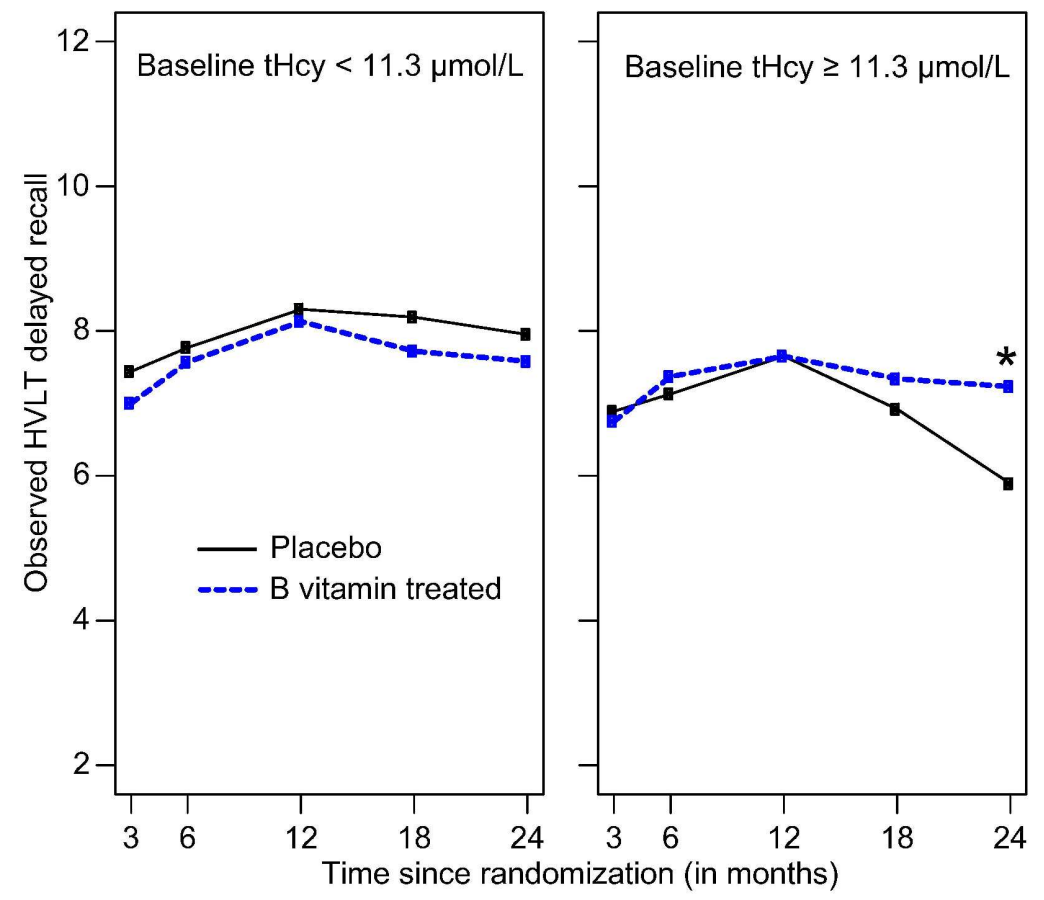

Effect of B vitamin treatment on a test of episodic memory.

The graphs illustrate the longitudinal effect of treatment on unadjusted mean HVLT-DR scores and its interaction with baseline tHcy level. 'Low' and 'High' baseline tHcy refer to values below and above the median $(11.3 \mu \mathrm{mol} / \mathrm{L})$, respectively. ${ }^{*} \mathrm{P}=0.001$ compared with placebo by logistic regression using GLMM (Table 4a).

\section{$177 \times 177 \mathrm{~mm}(600 \times 600 \mathrm{DPI})$}




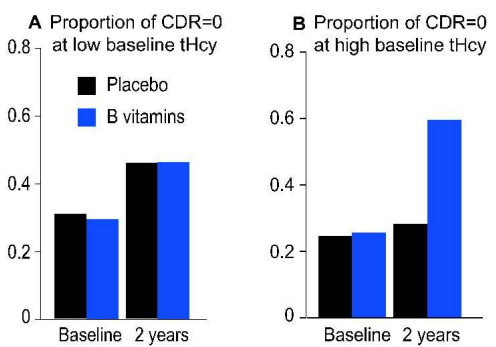

Effect of B vitamin treatment on the proportion of participants with a CDR score of zero. Low' and 'High' baseline tHcy refer to values below and above the upper quartile $(13.1 \mu \mathrm{mol} / \mathrm{L})$, respectively. $210 \times 297 \mathrm{~mm}(600 \times 600 \mathrm{DPI})$ 


\section{Supplementary Appendix for de Jager et al. B vitamin treatment in mild cognitive impairment: a randomised controlled trial}

\section{Supplementary Methods}

\section{Blood sampling and assays}

At baseline and after 24 months, blood samples were sent to a routine clinical laboratory for immediate determination of hematological and biochemical variables. In addition, serum, plasma samples containing EDTA and whole blood were processed and stored (Vogiatzoglou et al., 2008). Plasma tHcy was determined by fluorescence polarization immunoassay with the Abbott IMx ${ }^{\circledR}$ analyser (Shipchandler and Moore, 1995). Plasma folate and cobalamin concentrations were determined with microbiological assay(Molloy and Scott, 1997; Refsum et al., 2006). Genomic DNA was extracted from blood using the Wizard DNA Purification Kit (Promega, Southampton, UK). ApoE genotypes (NCBI Entrez Gene: 348) were determined using a one-stage PCR method (Wenham et al., 1991).

\section{Comparison of baseline values of those randomized with those who completed the trial}

To see if any bias was introduced by subjects who did not complete the trial, we have compared the baseline values in those who completed the trial $(\mathrm{N}=223)$ with the values in those who dropped out $(\mathrm{N}=$ 43). The results are shown in Table 1S.

\section{Cognitive tests}

TICS-M (Brandt et al., 1993)

The TICS-M is composed of 13 items that test the domains of orientation, memory, attention/calculation and language with a maximum possible score of 39. The TICS-M assigns a higher proportion of the total score to the memory component (56\%). Previous studies have shown it to be more effective than the MMSE, as it does not suffer from the same ceiling effects and scores are distributed normally, even in a sample of people with an on-average high IQ.(de Jager et al., 2003) We previously defined upper and lower MCI cut-off scores on the TICS-M which were applied to this trial for inclusion of participants with MCI (de Jager et al., 2003).

The Hopkins Verbal Learning Test-Revised (HVLT-R) (Brandt, 1991)

The HVLT consists of a 12-item word list, comprised of four words from each of three well-known semantic categories (e.g., 'precious stones', 'human shelter '; 'animals' in version 1 and other categories for the other 5 versions). Three learning trials are given and the score recorded each time. A total recall score is computed from the 3 trials out of 36 points and a learning index is calculated from the improvement from trial 1 to 3 . The HVLT-Revised includes a delayed recall trial for the 12 words administered after 15 to 20 minutes. Then, for yes/no recognition, from a list of 24 words, 12 original and 12 distractors, a 'Discrimination index' is calculated as (true positives-false positives). The HVLT is more sensitive to MCI than the MMSE (de Jager et al., 2009). In this paper we report delayed recall scores.

\section{Category Fluency (Morris et al., 1989)}

Fluency for naming items belonging to a specific category in 1 minute was used to assess semantic memory at both visits, and the total number of correct items was used as the score. During the telephone screening, animals were used, while at visits 1 and 2, we used 'fruits and vegetables'.

CLOX (Royall et al., 1998)

The subject is asked to draw a clock according to instruction on the back of a score sheet, through which a black circle is visible in the bottom right-hand corner (CLOX1). Once completed, the examiner draws the clock as required in front of the subject. The subject then has to copy the examiner's clock (CLOX2). Both parts are scored on 15 items for 15 points relating to various aspects of the task. For interpretation, the CLOX1 is an executive task requiring planning and execution by the subject with no help. The CLOX2 is a copying task to measure constructional praxis. 
We believe that the CLOX 2 - CLOX1 score used alone is inappropriate for assessment of cognitive performance. In fact, the difference score may mean different things for different subjects, depending on their CLOX1 score. As an example: if one subject has a CLOX $1=10$ and another a CLOX $1=12$ with both subjects scoring 1 point higher on CLOX 2 (i.e.11, 13), they would both have difference scores $($ CLOX 2-1 $)=1$. From this difference score the two subjects' performance is considered equal. However the second subject has better executive performance (since a CLOX1 of 12 is better than CLOX 1 of 10). Thus we decided to model CLOX1 score at visit 2 controlling for CLOX2 score at visit 2 (and also for the baseline CLOX1 score along with other confounders such as age etc.), as has recently been done in another study (McGuinness et al., 2010).

The Informant Questionnaire on Cognitive Decline in the Elderly (IQCODE) (Jorm, 2004) We used the shortened version which has 16 questions rated on a 5-point scale by an informant. The questions relate to whether the informant has seen a change in the subject from their previous state up to 10 years previously. The scale ranges from 'much improved' $=1$ to 'much worse' $=5$, with 'not much change' $=3$ as the midpoint. The questions rate memory and new learning ability as well as executive tasks such as decision making and problem solving, using mechanical appliances and handling finances. The maximum score totals 80 points and this is divided by the number of questions (16) to arrive at the final score.

The Clinical Dementia Rating (CDR) (Morris, 1993)

This is a scale used to assess the severity of dementia. The CDR assesses six domains of cognitive function with the subject and their study partner. These are memory, orientation, judgement and problem solving, community affairs, home and hobbies and personal care. All are rated on a 5-point scale, except personal care that has 4 points, and a sum of boxes score is obtained. The scores for the six areas are combined with an online algorithm available on the Alzheimer's Disease Research Centre (ADRC), Washington University website http://www.biostat.wustl.edu/adrc/ to rate severity of impairment. A global score of 0 equates with no dementia, 0.5 with MCI (may overlap with early AD), while 1,2 and 3 equate to mild, moderate and severe dementia, respectively. Our trial raters completed the ADRC online CDR training.

\section{Follow-up}

Participants were contacted by telephone at 3, 6, 9, 15 and 18 months after starting treatment to check compliance, adverse events and to administer the HVLT-R using the 6 different versions consecutively through the trial to reduce practice effects. After 24 months, participants returned to the clinic to repeat all tests including the cognitive test battery at baseline. The TICS-M was repeated at 12 and 27 months, i.e., 3 months after cessation of treatment, when participants were contacted by telephone to enquire about adverse events.

\section{Supplementary statistical information}

\section{Parameters for the GLM models in Table 4}

MMSE: Treatment category represents the logarithm of the odds ratio of a correct answer comparing B vitamins and placebo in the high tHcy group. In the high tHcy group, those treated with B vitamins are 1.58 times (i.e., $\exp (0.46))$ more likely to give a correct answer than those receiving placebo. Baseline tHcy represents the logarithm of the odds ratio of a correct answer comparing low and high tHcy placebo groups. Subjects receiving placebo in the low tHcy group are 1.63 times (i.e., $\exp (0.49)$ ) more likely to give a correct answer compared to subjects receiving placebo in the high tHcy group. Treatment $x$ tHcy represents the logarithm of the odds ratio of a correct answer comparing the B vitamins and placebo in the low tHcy group minus the logarithm of the odds ratio of a correct answer comparing the B vitamins and placebo in the high tHcy group.

Category fluency: Treatment category represents the logarithm of the ratio of the average category fluency comparing B vitamins and placebo in the high tHcy group. In the high tHcy group, the average 
number of words is $9.4 \%$ (i.e., $\exp (0.09)$ ) greater in those receiving B vitamins compared to placebo.

Baseline tHcy represents the logarithm of the ratio of the average category fluency between low and high tHcy placebo groups. Among subjects receiving placebo, the average number of words is $10 \%$ (i.e., $\exp (0.10))$ greater in low tHcy compared to high tHcy group. Treatment $x$ tHcy represents the logarithm of the ratio of the average category fluency between B vitamins and placebo in the low tHcy group minus the logarithm of the ratio of the average category fluency between B vitamins and placebo in the high tHcy group.

CDR: Treatment category represents the logarithm of the odds ratio of being normal (CDR $=0$ ) comparing B vitamins and placebo in the high tHcy group. In the high tHcy group, those receiving B vitamins are 5 times (i.e., $\exp (1.62)$ ) more likely to be normal than placebo. Baseline tHcy represents the logarithm of the odds ratio of being normal $(\mathrm{CDR}=0)$ comparing low and high tHcy placebo groups. Among subjects receiving placebo, those with low tHcy are 1.89 times (i.e., $\exp (0.64)$, not significant) more likely to be normal compared to those with high tHcy. Treatment $x$ tHcy represents the logarithm of the odds ratio of being normal $(\mathrm{CDR}=0)$ comparing $\mathrm{B}$ vitamins and placebo in the low tHcy group minus the logarithm of the odds ratio of being normal $(\mathrm{CDR}=0)$ comparing $\mathrm{B}$ vitamins and placebo in the high tHcy group.

IQCODE: Treatment category represents the difference in the average IQCODE between those receiving B vitamins and placebo in the high tHcy group. Baseline tHcy represents the difference in the average IQCODE between low and high tHcy placebo groups. Treatment $x$ tHcy represents the difference in the average IQCODE between those receiving B vitamins and placebo in the high tHcy group minus the difference in the average IQCODE between those receiving B vitamins and placebo in the low tHcy group.

CLOX: The final model obtained for CLOX1 at follow-up is the generalized linear model (GLM) with a binomial distribution and a logit link but with no interaction with the tHcy level at baseline. The model included CLOX1 score at visit 2 controlling for CLOX2 score at visit 2 and for baseline CLOX1 score (see above). The model specifies the probability of correctly answering a question at follow-up.

\section{Supplementary statistical tests}

Logistic regression using the GLMM was used to analyse the longitudinal data for the HVLT-DR tests. The same approach can be used for data collected at only two time points and we show the results of GLMM analysis for MMSE and category fluency (Figure 2S), for IQCODE (Figure 3S) and for the global CDR score (Figure 4S). This approach confirms the results of the GLM in the main text. For each test, B vitamin treatment had a beneficial effect in those participants with high baseline tHcy.

\section{Missing data}

For the GLM analyses, only subjects with final follow-up were used since the analysis is a crosssectional one. For the GLMM analyses, all the available data were used since this is a likelihood-based method and we found essentially the same results by analysis of the scores from completers only. 
Appendix Table 1S. Baseline Characteristics of the Study Participants

\begin{tabular}{|c|c|c|c|c|}
\hline Characteristic & $\begin{array}{c}\text { Randomized } \\
(\mathbf{N}=\mathbf{2 6 6})\end{array}$ & $\begin{array}{l}\text { Completed trial } \\
(\mathbf{N}=\mathbf{2 2 3})^{\mathrm{a}}\end{array}$ & $\begin{array}{c}\text { Dropped } \\
\text { out }(\mathrm{N}=43)^{\mathrm{b}}\end{array}$ & $\begin{array}{c}P \\
\text { value }^{\mathrm{a}, \mathrm{b}}\end{array}$ \\
\hline Age at baseline $-\mathrm{yr}$ & $76.8 \pm 4.9$ & $76.7 \pm 4.9$ & $77.0 \pm 4.8$ & 0.72 \\
\hline Female sex - N (\%) & $170(63.9 \%)$ & $143(64.1 \%)$ & $27(62.7 \%)$ & 0.96 \\
\hline Total education $-\mathrm{yr}$ & $14.5 \pm 3.4$ & $14.5 \pm 3.5$ & $14.3 \pm 2.8$ & 0.66 \\
\hline$A P O E \& 4$ carrier - N (\%) & $87(32.7 \%)$ & $69(30.9 \%)$ & $18(41.2 \%)$ & 0.16 \\
\hline Smoker - ever- N (\%) & $125(47.3 \%)$ & $104(46.8 \%)$ & $21(48.9 \%)$ & 0.71 \\
\hline $\operatorname{GDS}(0-30)$ \# & $6.7 \pm 4.7$ & $6.7 \pm 4.8$ & $7.0 \pm 4.7$ & 0.68 \\
\hline Hemoglobin* $-\mathrm{g} / \mathrm{dL}$ & $13.8 \pm 4.2$ & $13.8 \pm 1.2$ & $13.7 \pm 1.3$ & 0.54 \\
\hline $\mathrm{MCV}^{*}-\mathrm{fL}$ & $92.5 \pm 4.5$ & $92.7 \pm 4.2$ & $91.5 \pm 5.4$ & 0.16 \\
\hline Diabetes - ever - N (\%) & $20(7.5 \%)$ & $16(7.2 \%)$ & $4(9.3 \%)$ & 0.63 \\
\hline $\begin{array}{l}\text { Previous stroke, TIA, MRI } \\
\text { infarct }+\mathrm{N}(\%)\end{array}$ & $44(17.0 \%)$ & $40(18.0 \%)$ & $4(10.8 \%)$ & 0.28 \\
\hline Previous MI - N (\%) & $18(6.8 \%)$ & $17(7.6 \%)$ & $1(2.3 \%)$ & 0.21 \\
\hline Systolic BP $-\mathrm{mmHg}$ & $147 \pm 21$ & $147 \pm 22$ & $149 \pm 19$ & 0.37 \\
\hline Diastolic BP $-\mathrm{mmHg}$ & $80 \pm 11$ & $80 \pm 11$ & $82 \pm 10$ & 0.18 \\
\hline $\mathrm{BMI}-\mathrm{kg} / \mathrm{m}^{2}$ & $26.0 \pm 4.0$ & $25.9 \pm 3.9$ & $26.7 \pm 5.1$ & 0.31 \\
\hline Alcohol $\$$ - units/week & $8.0 \pm 8.5$ & $8.1 \pm 9.2$ & $7.4 \pm 7.5$ & 0.40 \\
\hline Creatinine $-\mu \mathrm{mol} / \mathrm{L}$ & $97 \pm 17$ & $97 \pm 17$ & $97 \pm 15$ & 0.81 \\
\hline TICS-M (0-39) & $24.9 \pm 2.8$ & $24.9 \pm 2.8$ & $24.4 \pm 3$ & 0.27 \\
\hline $\mathrm{CDR} \geq 0.5-\mathrm{N}(\%)$ & $188(70.9 \%)$ & $162(72.6 \%)$ & $26(60.5 \%)$ & 0.11 \\
\hline
\end{tabular}

Plus-minus values are means \pm SD. Student's t-test for comparisons of continuous variables and $\mathrm{Chi}^{2}$ for categorical variables, comparing those who completed the trial ${ }^{\mathrm{a}}$ with those dropped out ${ }^{\mathrm{b}}$. APOE, gene for apolipoprotein E; CDR, global clinical dementia rating; GDS, Geriatric Depression Scale; MCV, mean red cell volume; MI, myocardial infarct; TIA, transient ischemic attack; TICS-M, telephone interview of cognitive status, modified. *264 from randomized, 221 from completed; $† 259$ from randomized, 222 from completed; $\$ 220$ from randomized, 186 from completed, outlier omitted.

Appendix Table 1S contd. Baseline Characteristics of the Study Participants: vitamins, etc.

\begin{tabular}{|l|c|c|c|c|c|c|c|}
\hline \multicolumn{1}{|c|}{ Characteristic } & \multicolumn{2}{|c|}{ Randomized (N= 266) } & \multicolumn{2}{|c|}{$\begin{array}{c}\text { Completed trial } \\
(\mathbf{N = 2 2 3})^{\mathbf{a}}\end{array}$} & \multicolumn{2}{|c|}{ Dropped out (N=43) } & \multicolumn{2}{|c|}{$\begin{array}{c}\text { P } \\
\text { value }^{\mathbf{a}, \mathbf{b}}\end{array}$} \\
\hline & $\begin{array}{c}\text { Geometric } \\
\text { mean }\end{array}$ & $\mathbf{9 5 \%}$ CI & $\begin{array}{c}\text { Geometric } \\
\text { mean }\end{array}$ & $\mathbf{9 5 \%}$ CI & $\begin{array}{c}\text { Geometric } \\
\text { mean }\end{array}$ & $\mathbf{9 5 \%}$ CI & \\
\hline tHcy $-\mu \mathrm{mol} / \mathrm{L}$ & 11.5 & $11.1-11.9$ & 11.4 & $11.0-11.9$ & 11.7 & $10.7-12.8$ & 0.61 \\
\hline Folate $-\mathrm{nmol} / \mathrm{L}$ & 22.2 & $20.6-24.0$ & 22.9 & $20.5-24.9$ & 19.4 & $15.9-23.5$ & 0.15 \\
\hline Vitamin $\mathrm{B}_{12}$ & 326 & $312-342$ & 328 & $313-343$ & 320 & $286-358$ & 0.71 \\
\hline HoloTC $-\mathrm{pmol} / \mathrm{L}$ & 66 & $62-71$ & 64 & $59-70$ & 76 & $65-89$ & 0.06 \\
\hline
\end{tabular}

Log transformed values were used for the calculations. 
Appendix Table 2S. Number of participants with overall CDR rating of 0 or $\geq 0.5$ at baseline and followup for placebo and B vitamin treatment groups, split by low and high (upper quartile) baseline tHcy levels.

\begin{tabular}{|c|c|c|c|c|c|}
\hline & \multirow[t]{2}{*}{ CDR } & \multicolumn{2}{|c|}{ Placebo group } & \multicolumn{2}{|c|}{ B vitamin group } \\
\hline & & Baseline & Follow-up & Baseline & Follow-up \\
\hline \multirow[t]{2}{*}{ Low tHcy $(<13.1)$} & 0 & 22 & 33 & 21 & 34 \\
\hline & $\geq 0.5$ & 48 & 37 & 51 & 38 \\
\hline \multirow[t]{2}{*}{ High tHcy $(\geq 13.1)$} & 0 & 6 & 7 & 6 & 14 \\
\hline & $\geq 0.5$ & 19 & 18 & 18 & 10 \\
\hline
\end{tabular}

The table only includes participants who had both baseline and follow-up CDR assessments ( $\mathrm{N}=95$ in placebo group; $\mathrm{N}=96$ in $\mathrm{B}$ vitamin group). Some participants had study partners who withdrew during the study and so have no CDR at follow-up ( $=32$; of whom 17 were taking placebo and 15 taking $\mathrm{B}$ vitamins). Comparison of the demographic variables at baseline listed in Table 1S between those had a CDR at follow-up and those who did not showed no significant differences, except that there was a lower proportion of APOE $\varepsilon 4$ carriers (14.7\%) in those who dropped out compared with those who remained $(33.5 \%, \mathrm{P}=0.02$ Fisher $)$ 


\section{Figure1S. CONSORT diagram showing participant flow in the trial}

\section{Figure2S. Effect of B vitamin treatment on cognitive test scores}

The graphs illustrate the longitudinal effect of treatment on cognitive scores and its interaction with baseline tHcy level using generalized linear mixed effect models. 'Low' and 'High' baseline tHcy refer to values below and above the median $(11.3 \mu \mathrm{mol} / \mathrm{L})$, respectively. A-C, MMSE; D-E, category fluency.

\section{Figure 3S. Effect of B vitamin treatment on the IQCODE}

The graphs illustrate the longitudinal effect of treatment on the mean IQCODE and its interaction with baseline tHcy level using GLMM (Guassian distribution, i.e. a linear mixed effects model). A shows the pattern over time in participants with baseline tHcy below the top quartile $(13.1 \mu \mathrm{mol} / \mathrm{L})$ and $\mathbf{B}$ shows the pattern in those in the top quartile. In the 'high tHcy subjects' the estimated slope for the placebo group was 0.02 and for the $\mathrm{B}$ vitamin group was -0.09 . The difference between the two slopes is highly significant $(\mathrm{P}=0.006)$.

\section{Figure 4S. Effect of $B$ vitamin treatment on clinical dementia rating}

The graphs illustrate the longitudinal effect of treatment on global CDR score and its interaction with baseline tHcy level using GLMM. A and B show the pattern, over time, of the model-estimated proportion of subjects with a CDR score of zero over time. Low baseline tHcy is $<13.1 \mu \mathrm{mol} / \mathrm{L}$; high baseline tHcy is $\geq 13.1 \mu \mathrm{mol} / \mathrm{L}$. C shows the pattern, over time of the model-estimated odds ratio of a CDR score of zero for a person with high tHcy on B vitamin treatment compared with the same person on placebo. For the actual numbers with CDR of zero see Table $2 \mathrm{~S}$. 


\section{References}

Brandt J 1991 The Hopkins Verbal Learning Test: Development of a new memory test with six equivalent forms. Clinical Neuropsychologist 5: 125-142.

Brandt J, Welsh KA, Breitner JC, Folstein MF, Helms M \& Christian JC 1993 Hereditary influences on cognitive functioning in older men. A study of 4000 twin pairs. Arch Neurol 50: 599-603.

de Jager CA, Budge MM \& Clarke R 2003 Utility of TICS-M for the assessment of cognitive function in older adults. Int J Geriatr Psychiatry 18: 318-324.

de Jager CA, Schrijnemaekers AC, Honey TE \& Budge MM 2009 Detection of MCI in the clinic: evaluation of the sensitivity and specificity of a computerised test battery, the Hopkins Verbal Learning Test and the MMSE. Age Ageing 38: 455-460.

Jorm AF 2004 The Informant Questionnaire on cognitive decline in the elderly (IQCODE): a review. Int Psychogeriatr 16: 275-293.

McGuinness B, Barrett SL, Craig D, Lawson J \& Passmore AP 2010 Executive functioning in Alzheimer's disease and vascular dementia. Int J Geriatr Psychiatry 25: 562-568.

Molloy AM \& Scott JM 1997 Microbiological assay for serum, plasma, and red cell folate using cryopreserved, microtiter plate method. Methods Enzymol 281: 43-53.

Morris JC 1993 The Clinical Dementia Rating (CDR): current version and scoring rules. Neurology 43: $2412-2414$.

Morris JC, Heyman A, Mohs RC, Hughes JP, van Belle G, Fillenbaum G, Mellits ED \& Clark C 1989 The Consortium to Establish a Registry for Alzheimer's Disease (CERAD). Part I. Clinical and neuropsychological assessment of Alzheimer's disease. Neurology 39: 1159-1165.

Refsum H, Johnston C, Guttormsen AB \& Nexo E 2006 Holotranscobalamin and total transcobalamin in human plasma: determination, determinants, and reference values in healthy adults. Clin Chem 52: 129-137.

Royall DR, Cordes JA \& Polk M 1998 CLOX: an executive clock drawing task. J Neurol Neurosurg Psychiatry 64: 588-594.

Shipchandler MT \& Moore EG 1995 Rapid, fully automated measurement of plasma homocyst(e)ine with the Abbott IMx analyzer. Clin Chem 41: 991-994.

Vogiatzoglou A, Refsum H, Johnston C, Smith SM, Bradley KM, de Jager C, Budge MM \& Smith AD 2008 Vitamin B12 status and rate of brain volume loss in community-dwelling elderly. Neurology 71: 826-832.

Wenham PR, Price WH \& Blandell G 1991 Apolipoprotein E genotyping by one-stage PCR. Lancet 337: 11581159. 


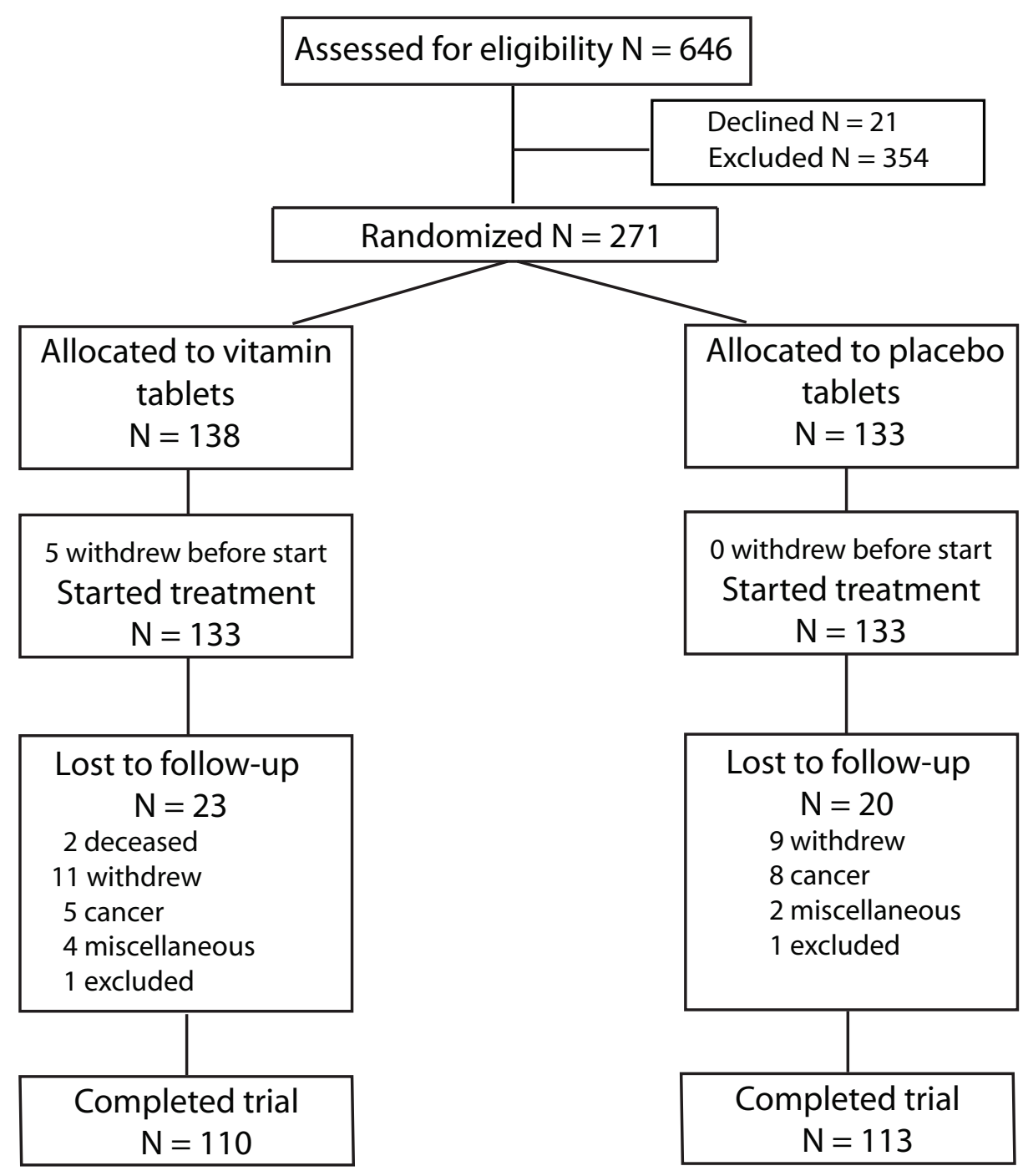

Figure 1S 
A. Global function

with low baseline tHcy
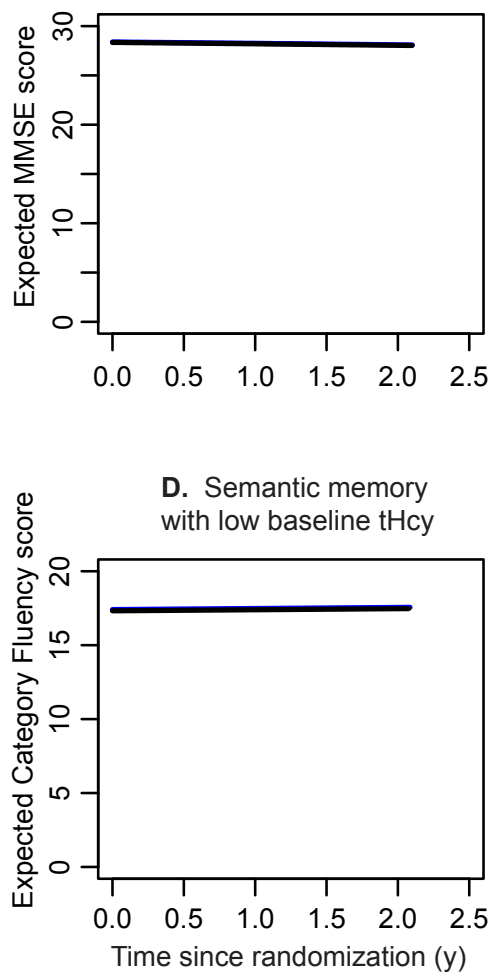

B. Global function with high baseline tHcy

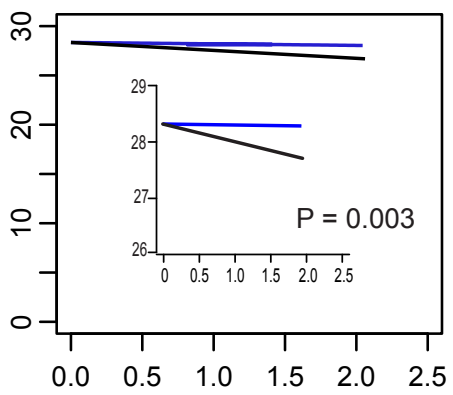

E. Semantic memory with high baseline tHcy

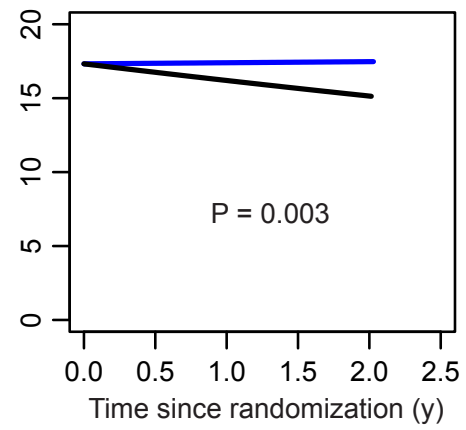

C. Global function with high baseline tHcy: odds ratio of right answer, treated vs. placebo
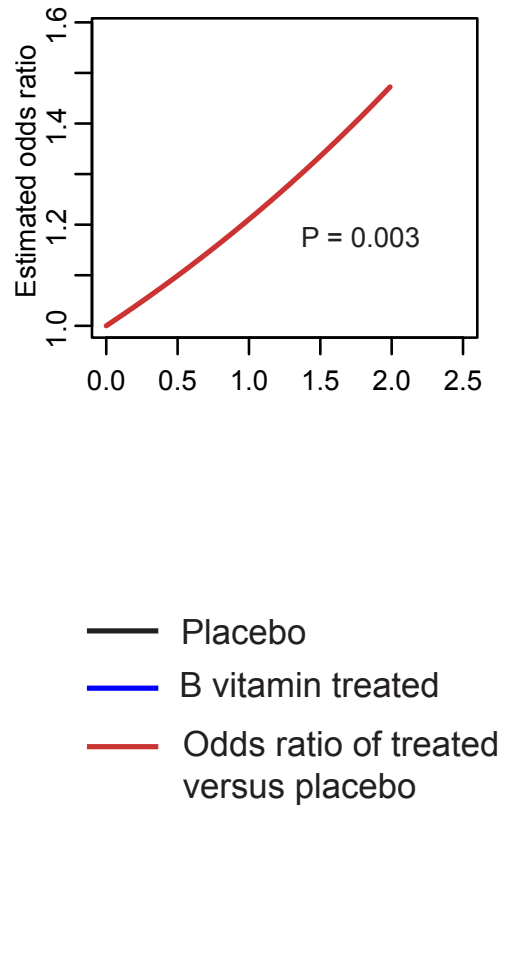

Figure 2S 

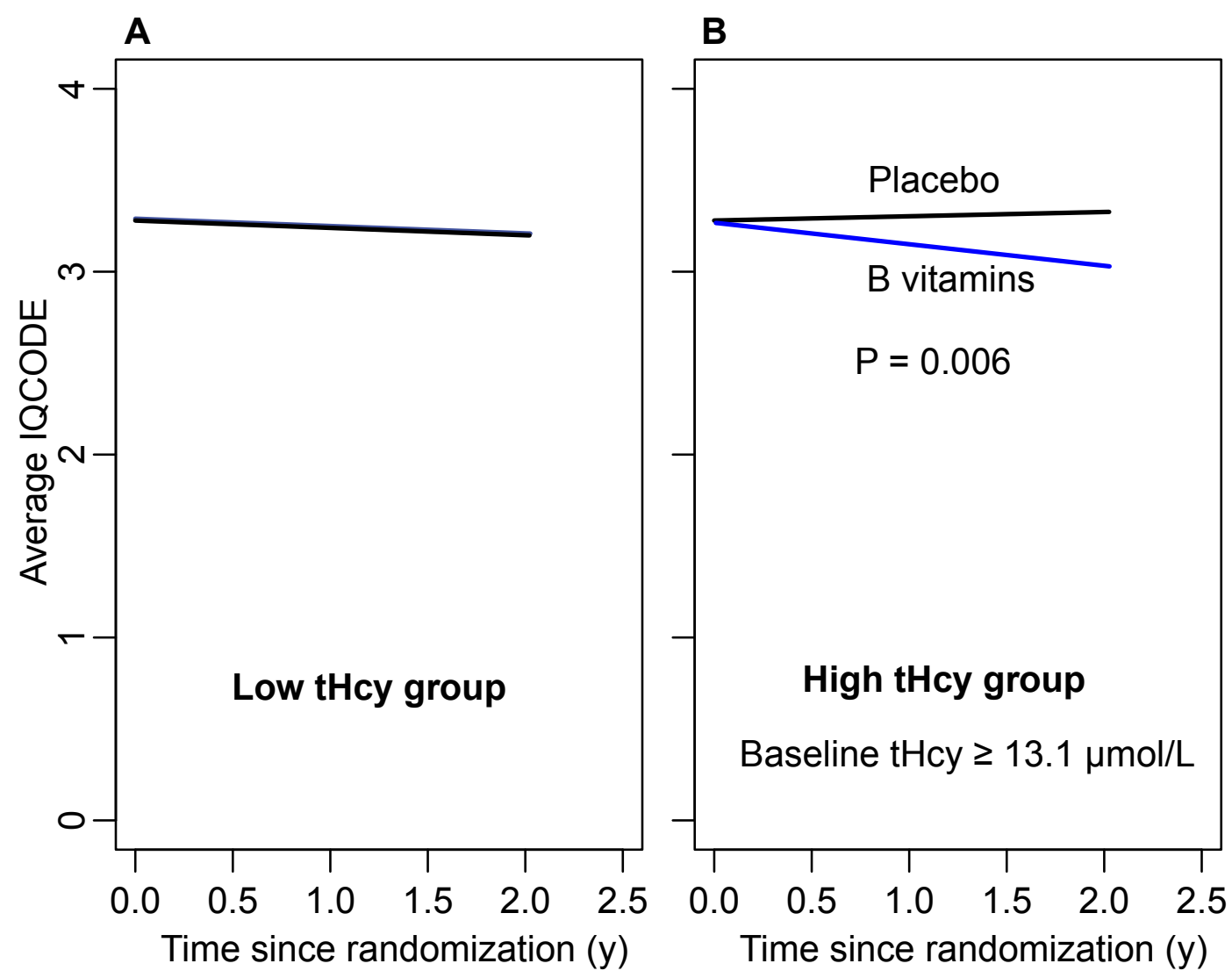

Figure 3S 

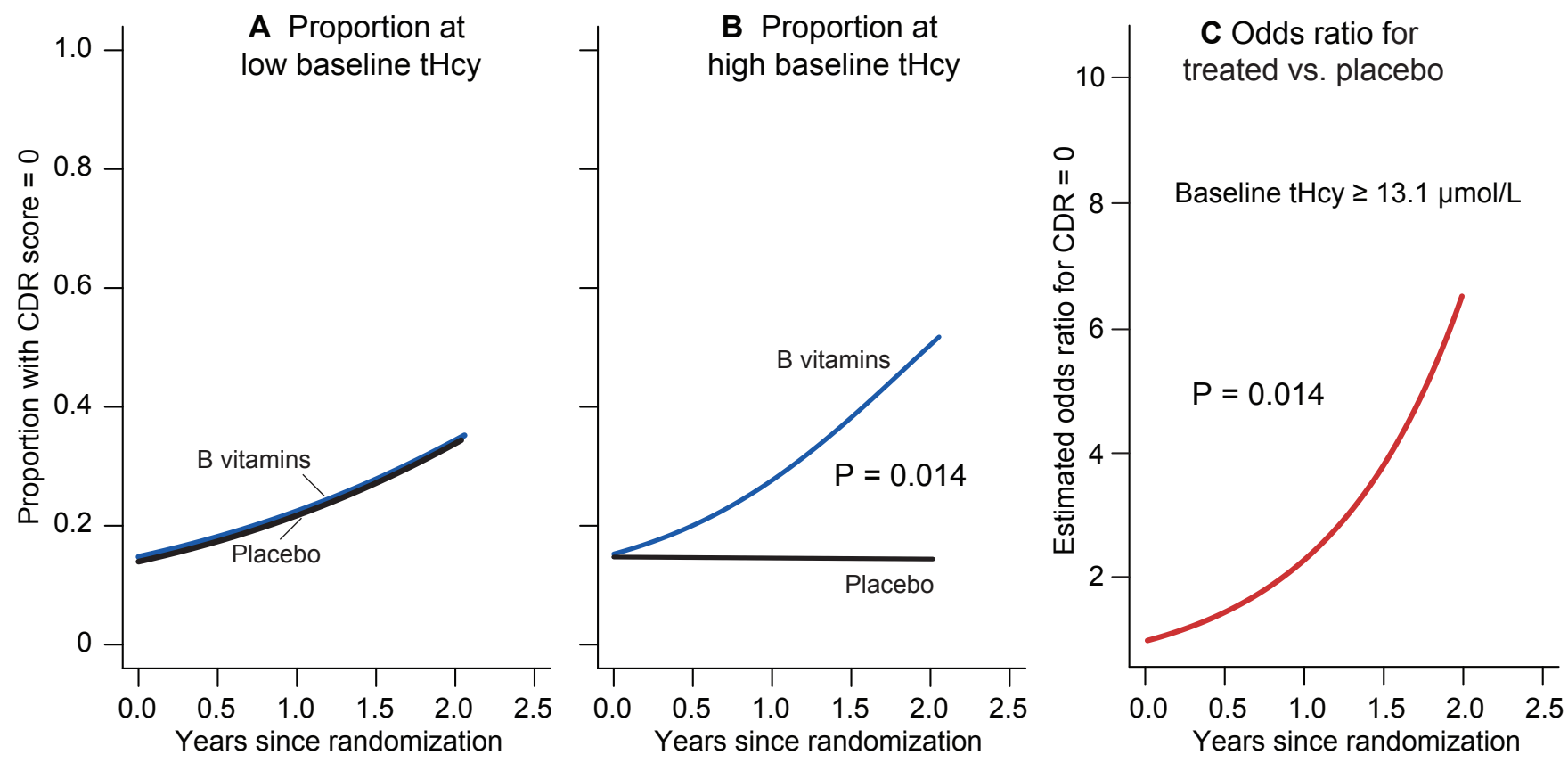

Figure 4S 